\title{
EVAPOTRANSPIRAÇÃO DE REFERÊNCIA: UMA ABORDAGEM ATUAL DE DIFERENTES MÉTODOS DE ESTIMATIVA ${ }^{1}$
}

\author{
Luiz Gonsaga de Carvalho², Gervásio Fernando Alves Rios², Wezer Lismar Miranda², Pedro Castro Neto ${ }^{2}$
}

\section{ABSTRACT \\ REFERENCE EVAPOTRANSPIRATION: CURRENT ANALYSIS OF DIFFERENT ESTIMATING METHODS}

The reference evapotranspiration (ETo), originally known as potential evapotranspiration (ETp), is an important agrometeorological parameter, mainly for planning and managing irrigation, besides being considered a climatic element of hydric demand, and that is the reason it is used in meteorological, climatological, and hydrological studies. There are several methods for estimating ETo, from the simpler ones, purely empiric, to those which present a consistent basis of the phenomenon, however, some empiricism is always present. This diversity of methods happens due to the complexity of the water transfering from the soil-plant system to the atmosphere, different climates around the world, and difficulties for obtaining the necessary meteorological data to make those methods work. The Penman-Monteith-FAO (PM-FAO) is internationally known as the most appropriate approach for estimating ETo, being adopted as standard two decades ago. Besides representing, in a consistent way, the evapotranspiration biophysical phenomenon, it makes use of almost all meteorological data observed in surface weather stations. However, when it is not possible to apply this method, it is necessary to investigate which method would be the most suitable for local conditions. Therefore, this study was carried out in order to present, discuss, and point out the most appropriate method(s) for each specific use.

KEY-WORDS: FAO Penman-Monteith; potential evapotranspiration; evapotranspiration estimating; ETo estimating methods.

\section{INTRODUÇÃO}

As atividades agrícolas demandam grandes quantidades de água e, sendo cada vez mais preocupante a sua escassez, esforços têm sido empregados no desenvolvimento de pesquisas que possibilitem a sua economia, em todo o planeta. Neste sentido, o correto conhecimento da evapotranspiração das

\section{RESUMO}

A evapotranspiração de referência (ETo), originalmente introduzida sob o termo evapotranspiração potencial (ETp), é um importante parâmetro agrometeorológico, principalmente para planejamento e manejo de irrigação, mas, também, é considerada elemento climático de demanda hídrica, daí sua aplicação em estudos meteorológicos, climatológicos e hidrológicos. Existem diversos métodos para a estimativa da ETo, desde aqueles mais simples, com base puramente empírica, até aqueles que apresentam embasamento físico consistente sobre o fenômeno, contudo, há sempre algum empirismo presente. Esta diversidade de métodos ocorre devido à complexidade da transferência de água do sistema solo-planta para a atmosfera, aos diferentes climas presentes no mundo e à dificuldade de obtenção dos elementos meteorológicos necessários para a alimentação destes métodos. O método Penman-Monteith-FAO (PM-FAO) é considerado, internacionalmente, o mais apropriado para a estimativa da ETo, sendo adotado como padrão e que já vem sendo usado há duas décadas. Este método, além de procurar representar, de maneira consistente, o fenômeno biofísico da evapotranspiração, é alimentado por quase todos os elementos meteorológicos observados em estações meteorológicas de superfície. Porém, quando há impossibilidade da aplicação deste método, é necessário averiguar qual o método mais indicado, diante das condições locais. Assim, este estudo teve por propósitos apresentar, discutir e sinalizar o(s) método(s) mais adequado(s), diante da realidade de uso.

PALAVRAS-CHAVE: Penman-Monteith-FAO; evapotranspiração potencial; estimativa de evapotranspiração; ETo - métodos de estimativa.

culturas, seja para projeto e/ou manejo de irrigação, assume fundamental importância. Uma das alternativas para se racionalizar o uso da água, em projetos agrícolas, é estimar a evapotranspiração da cultura (ETc), a partir da evapotranspiração de referência (ETo) e do coeficiente de cultura (Kc).

O termo "evapotranspiração" (ET) foi introduzido por Thornthwaite \& Wilm (1944) e, ao

1. Trabalho recebido em dez./2010 e aceito para publicação em jun./2011 (nº registro: PAT 12760/ DOI: 10.5216/pat.v41i3.12760).

2. Universidade Federal de Lavras, Departamento de Engenharia, Lavras, MG, Brasil.

E-mails: lgonsaga@deg.ufla.br, gervasiorios@yahoo.com.br,wmlismar@yahoo.com.br, pedro@oleo.ufla.br. 
final da década de 1940, surgiram duas importantes contribuições científicas, não somente para a agricultura, mas, também, para a climatologia e hidrologia: "An approach towards a rational classification of climate", de Warren Thornthwaite (1948) e "Natural evaporation from open water, bare soils and grass", de Howard Penman (1948).

Camargo \& Camargo (2000) classificaram da seguinte forma o trabalho destes dois pesquisadores: o trabalho de Thornthwaite foi uma pesquisa analítica, empírica e fundamentada na análise dos fatos, enquanto Penman fez uma abordagem sintética, científica e firmada no conhecimento físico que rege o fenômeno.

De acordo com Sediyama (1996), Thornthwaite tinha a preocupação de explicar as variações sazonais do balanço de água no solo e tentar definir as diferenças regionais do clima. Por esta razão, este método é apenas uma função da temperatura média do ar e da duração do dia, em média mensal. Penman preocupou-se com os processos físicos envolvidos na evaporação e com o desenvolvimento de um método, que, a partir de elementos meteorológicos, expressasse a estimativa da taxa de evaporação da água em contato livre com a atmosfera, da umidade da superfície do solo e da vegetação.

A partir destes trabalhos, aparece o conceito de evapotranspiração potencial (ETp), caracterizada como a transferência de água do sistema solo-planta para a atmosfera, sob condições padronizadas, ou seja, área com extensa superfície natural, totalmente coberta por vegetação baixa, de altura uniforme e com elevado índice de área foliar (IAF), de crescimento ativo na fase adulta (a grama é a principal vegetação adotada, e, em alguns tipos de clima, adota-se a alfafa) e teor de água do solo próximo ou na capacidade de campo. Sob estas condições de contorno, e considerando-as invariáveis no tempo e espaço, a transferência de água do sistema solo-planta para a atmosfera (evapotranspiração) ocorre como função apenas do balanço vertical de energia, ou seja, das condições atmosféricas sobre a vegetação, sem interferências advectivas, podendo ser estimada por métodos modelados sob embasamentos matemáticos teórico-empíricos desenvolvidos e testados para várias condições climáticas.

Mais tarde, na década de 1960, Monteith, com base no método de Penman, propôs um novo método que estimava diretamente a ETc, denominando-o de Penman-Monteith, e Doorenbos \& Pruitt (1977) introduziram o conceito de evapotranspiração de referência (ETo), em substituição ao termo ETp. Mas foi em 1990 que houve uma grande revolução sobre a proposição da conceituação e estimativa da ETo. Neste ano, no período de 28 a 31 de maio, a Food and Agriculture Organization (FAO) promoveu, em Roma, Itália, um encontro de especialistas na área, para atender a vários objetivos, dentre eles o de analisar os conceitos e procedimentos de metodologias de cálculos da ET, com enfoque no estabelecimento de uma nova definição para a cultura de referência e o método que pudesse estimar a ET, para esta referência (Pereira et al. 1997). Assim, o novo conceito proposto para a ETp passou a ser, de fato, a ETo, tornando-se, este conceito, desde então, largamente utilizado, e o método recomendado para sua estimativa foi o desenvolvido por Penman-Monteith, que, após parametrização, passou a denominar-se Penman-Monteith FAO (PM-FAO), o qual foi bastante aceito, internacionalmente. Neste caso, a cultura de referência utilizada é uma cultura hipotética, cujas características se assemelham, bem de perto, à ET da grama. Isto permite que tais características (valores numéricos) mantenham-se como parâmetros constantes adotados no cálculo da ETo. Estes parâmetros, para a cultura hipotética, são: altura de $0,12 \mathrm{~m}$, albedo igual a 0,23 e resistência da cultura ao transporte de vapor d'água igual a $69 \mathrm{~s} \mathrm{~m}^{-1}$. Logo, a ETo é um elemento indicativo da demanda hídrica das culturas de um determinado local e período.

Apesar da proposição da FAO, ainda é bastante comum o uso dos termos ETp e ETo. Pode-se inferir que, para estudos climatológicos, o termo ETp continue sendo o mais utilizado, pois, quase sempre, está ligado à análise do potencial hídrico de uma região, enquanto a ETo é bastante adequada para projetos e manejo de irrigação, uma vez que a ETc, normalmente, é determinada em duas etapas, aplicando-se o coeficiente de ajuste Kc à evapotranspiração da cultura de referência (ETo), que, no caso, é a cultura hipotética.

Durante todos esses anos, muitos métodos foram desenvolvidos para estimativa da ETo. Isto ocorre em razão de três importantes situações: adequação do método às condições climáticas da região, simplicidade de uso e limitação de elementos meteorológicos ou climáticos que alimentam estes métodos. Devido à diversidade destes métodos, em razão da grande variabilidade dos parâmetros que influenciam o fenômeno, e também ao empirismo, 
em muitos deles, é comum haver estudos utilizando artifícios estatísticos para comparar os métodos e, assim, avaliar aquele que tem maior aplicabilidade ao local de estudo. Portanto, este artigo apresenta uma revisão fundamentada nos avanços da modelagem da ETo, na última década, propondo apresentar os resultados de desempenhos dos diversos métodos e as possibilidades e limitações de uso, complementados por uma visão crítica, para responder à seguinte pergunta: "Qual o melhor método a ser aplicado?".

\section{MÉTODOS DE ESTIMATIVA DA EVAPOTRANSPIRAÇÃO DE REFERÊNCIA}

Em razão da grande quantidade de métodos que possibilitam estimar a ETo, são discutidos, neste trabalho, aqueles mais comuns, encontrados na literatura, e, mais especificamente, os métodos baseados em equações empíricas e/ou com fundamentação física. Muitos destes métodos possuem variantes, por questões de ajustes e calibrações locais, elevando, ainda mais, a quantidade de métodos disponíveis. Por isto, está representada, na Tabela 1, uma síntese destes métodos. Ressalta-se que as referências originais dos respectivos métodos poderão ser encontradas nas referências citadas.

Além dos métodos matemáticos apresentados anteriormente, atualmente, muitos pesquisadores têm estudado a aplicação de técnicas para a estimativa da ETo, até então aplicadas, especificamente, a outras áreas do conhecimento. Neste sentido, verifica-se a aplicação de redes neurais em trabalhos de Chauhan \& Shrivastava (2009) e Kumar et al. (2010). Além destes, para efeito de planejamento de uso do solo, existem estimativas de ETo efetuadas com base em imagens de satélites, tais como as apresentadas em trabalhos desenvolvidos por Bezerra et al. (2008) e Folhes et al. (2009).

\section{DESEMPENHO DOS MÉTODOS DE ESTIMATIVA DA ETo}

Conforme mencionado anteriormente, o método de Penman-Monteith-FAO (PM-FAO) foi um aperfeiçoamento do método original de Penman (PEN), e diversos estudos, no Brasil e no mundo, têm comprovado que o método PM-FAO é bastante preciso (Xu \& Chen 2005, Yoder et al. 2005, López-Urrea et al. 2006, Jabloun \& Sahli 2008, Barros et al. 2009), sendo, por esta razão, bastante utilizado como padrão de comparação com os outros métodos. Alguns estudos apresentam variantes do método PM-FAO, buscando melhores ajustes para determinadas regiões, tal como ocorre com o método PM-ASCE (Temesgen et al. 2005, Allen et al. 2006, Gavilán et al. 2007, Gavilán et al. 2008, Suleiman \& Hoogenboom 2009). Gavilán et al. (2007), no sul da Espanha, próximo a Córdoba, concluíram que o método ASCE-PM, na base horária, melhorou a precisão da estimativa da ETo, se comparado ao método PM-FAO, corroborando resultados apresentados por Jensen et al. (1990).

Em trabalho realizado por Sentelhas et al. (2010), para doze localidades no sul de Ontário, Canadá, estudando métodos que utilizam, basicamente, somente dados de temperaturas, como o PT, HG e TW, e o método alternativo PM-FAO, que utiliza apenas temperaturas máximas e mínimas (Allen et al. 1998), observou-se que os métodos HG e TW, após alguns ajustes, foram as melhores opções para estimar a ETo. Trabalhos desta natureza evidenciam que a calibração de alguns parâmetros dos métodos pode melhorá-los consideravelmente, para a estimativa da ETo. Semelhantemente, aplicando-se o método PM-FAO alternativo e utilizando-se apenas temperaturas máximas e mínimas, os trabalhos desenvolvidos por Jabloun \& Sahli (2008), na Tunísia, e Silva et al. (2008), em Lavras (MG), mostraram a viabilidade deste método para os locais estudados, quando faltam dados para entrada ao método PM-FAO.

Sentelhas et al. (2008) aplicaram o método PM-FAO no desenvolvimento do balanço hídrico climatológico de Thornthwaite \& Mather (1955), para todo o Brasil, considerando o PM-FAO o mais representativo para a estimativa da demanda de evapotranspiração (ETp). O mesmo procedimento foi utilizado por Carvalho et al. (2008), no zoneamento ecológico-econômico, para o Estado de Minas Gerais, destacando que os parâmetros geoestatísticos indicaram melhor qualidade na interpolação dos resultados obtidos com a estimativa da ETp pelo método PM-FAO, quando comparado ao cálculo da ETp, pelo método TW.

Doorembos \& Pruitt (1977) advertem que o uso de dados climáticos médios, em métodos combinados, ou seja, envolvendo os componentes aerodinâmico e radiativo, como é o caso de PM-FAO e PEN, pode conduzir a erros significativos, resultantes das combinações entre os elementos climáticos. O método PM-FAO pode, também, variar bastante, quanto à questão 
Tabela 1. Métodos de estimativa da evapotranspiração de referência.

\begin{tabular}{|c|c|c|c|c|}
\hline Método & $\begin{array}{l}\text { Referência original } \\
\text { e/ou citada }\end{array}$ & $\begin{array}{l}\text { Elementos } \\
\text { meteorológicos } \\
\text { de entrada }^{1}\end{array}$ & $\begin{array}{l}\text { Variáveis } \\
\text { auxiliares } \\
\text { de entrada }\end{array}$ & Observações ${ }^{2}$ \\
\hline Penman (PEN) & $\begin{array}{l}\text { Penman (1948), } \\
\text { Yoder et al. (2005) }\end{array}$ & $\begin{array}{l}\text { Tx, Tn, T, UR, } \\
\text { v, n, P, Rs, Rn }\end{array}$ & DJ, Lat & Conhecido também por Penman Original. \\
\hline $\begin{array}{l}\text { Thornthwaite } \\
\text { (TW) }\end{array}$ & Thornthwaite (1948) & Tmn, Tm, T & Lat & $\begin{array}{l}\left.\text { Estimativa da ETp, em escala mensal ( } \mathrm{mm} \text { mês }{ }^{-1}\right) \text {. Utilizando-se T, pode-se } \\
\text { estimar a ETp ou ETo, em escala diária, desde que o resultado seja dividido } \\
\text { pelo número de dias do respectivo mês - este critério pode ser válido para } \\
\text { todos os métodos cujo resultado seja apresentado em mm mês }{ }^{-1} \text {. }\end{array}$ \\
\hline
\end{tabular}

\begin{tabular}{|c|c|c|c|c|}
\hline Turc (TC) & $\begin{array}{l}\text { Yoder et al. }(2005), \\
\text { Trajkovic \& Kolakovic } \\
\text { (2009), Tabari (2010) }\end{array}$ & T, UR, Rs & DJ, Lat & - \\
\hline $\begin{array}{l}\text { Penman-Monteith- } \\
\text { FAO (PM-FAO) }\end{array}$ & Allen et al. (1998) & $\begin{array}{l}\text { Tx, Tn, T, UR, } \\
\text { v, n, P, Rs, Rn }\end{array}$ & DJ, Lat & Também conhecido por Penman-Monteith FAO 56. \\
\hline $\begin{array}{l}\text { Tanque Classe A } \\
\text { (TCA) }\end{array}$ & $\begin{array}{l}\text { Doorenbos \& Pruitt } \\
\text { (1977), Allen et al. } \\
\text { (1998), Barros et al. } \\
\text { (2009) }\end{array}$ & ECA, v, UR & $\begin{array}{l}\text { Extensão } \\
\text { da } \\
\text { bordadura } \\
\text { do tanque }\end{array}$ & - \\
\hline
\end{tabular}

\begin{tabular}{|c|c|c|c|c|}
\hline $\begin{array}{l}\text { Blaney-Criddle } \\
(\mathrm{BC})\end{array}$ & $\begin{array}{l}\text { Doorenbos \& Pruitt } \\
\text { (1977), Pereira et al. } \\
\text { (1997), López-Urrea et } \\
\text { al. (2006) }\end{array}$ & $\begin{array}{l}\text { Tm, URmín, } \\
\text { n, v }\end{array}$ & DJ, Lat & $\begin{array}{l}\text { Também conhecido por Blaney-Criddle FAO 24. Todos os dados de entrada } \\
\left.\text { em médias mensais. Estimativa da ETo em escala mensal (mm mês }{ }^{-1}\right) \text {. Com } \\
\text { DJ e Lat, pode-se estimar o parâmetro "p" sem uso de tabela. Ver, também, } \\
\text { observaçấo do método TW, para estimativa da ETo em escala diária. }\end{array}$ \\
\hline Makkink (MK) & $\begin{array}{l}\text { Pereira et al. (1997), } \\
\text { Yoder et al. (2005) }\end{array}$ & Rs, P, T & DJ, Lat & $\begin{array}{l}\text { Os parâmetros "s" ou " } \Delta \text { ", } \gamma \text { e } \lambda \text { são estimados da mesma forma que no } \\
\text { método PM-FAO. }\end{array}$ \\
\hline Hamon (HM) & $\begin{array}{l}\text { Borges \& Mendiondo } \\
(2007) \text {, Pereira et al. } \\
(2009)\end{array}$ & $\mathrm{T}$ & DJ, Lat & DJ e Lat para estimativa de N (duração astronômica do dia). \\
\hline
\end{tabular}

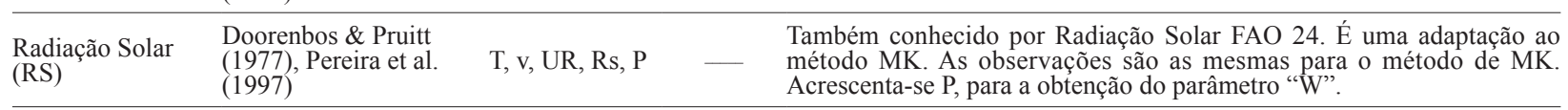

(1997) Pereira et al. T, v, UR, Rs, P

Linacre (LN) Pereira et al. (1997) $\quad \begin{aligned} & \text { Tn } \\ & \text { Tn Tpo, Tx, Lat, Alt }\end{aligned}$

Trata-se de uma simplificação do método PEN. A parte (Tm-Tpo) deste método pode ser estimada em função de Alt, Tm, Tx, Tn e, ainda, pela diferença entre as temperaturas médias dos meses mais quente e mais frio. Estima a ETo em escala mensal $\left(\mathrm{mm}^{\mathrm{m}} \mathrm{s}^{-1}{ }^{-1}\right.$. Ver, também, observação do método TW, para estimativa da ETo em escala diária.

\begin{tabular}{|c|c|c|c|c|}
\hline Camargo (CAM) & Pereira et al. (1997) & $\mathrm{Tm}$ & DJ, Lat & $\begin{array}{l}\text { É uma simplificação do método TW. Estima a ETp em período de } 10 \text { ou } \\
30 \text { dias. Ver, também, observação do método TW, para estimativa da ETo } \\
\text { em escala diária. }\end{array}$ \\
\hline Jensen-Haise (JH) & $\begin{array}{l}\text { Pereira et al. (1997), } \\
\text { Trajkovic \& Kolakovic } \\
\text { (2009) }\end{array}$ & Rs, T & DJ, Lat & - \\
\hline Hargreaves (HG) & $\begin{array}{l}\text { Allen et al. (1998), } \\
\text { Sousa et al. (2010) }\end{array}$ & $\mathrm{T}, \mathrm{Tx}, \mathrm{Tn}$ & DJ, Lat & Bastante divulgado como método "Hargreaves e Samani". \\
\hline $\begin{array}{l}\text { Priestley-Taylor } \\
\text { (PT) }\end{array}$ & $\begin{array}{l}\text { Pereira et al. (1997), } \\
\text { Sentelhas et al. (2010), } \\
\text { Tabari (2010) }\end{array}$ & T, P, Rn, G & DJ, Lat & $\begin{array}{l}\text { É uma simplificação do método PEN. Rn e G podem seguir os mesmos } \\
\text { procedimentos do método PM-FAO. }\end{array}$ \\
\hline Holdridge (HD) & Vega \& Jara (2009) & $\mathrm{T}$ & & $\begin{array}{l}\mathrm{O} \text { parâmetro " } \mathrm{C}_{\mathrm{HO}} \text { " deste método permite ajustar as estimativas em } \\
\text { diferentes escalas de tempo (diária, mensal e anual). }\end{array}$ \\
\hline Kharrufa (KF) & $\begin{array}{l}\text { Borges \& Mendiondo } \\
\text { (2007), Pereira et al. } \\
(2009)\end{array}$ & $\mathrm{T}$ & DJ, Lat & DJ e Lat para estimativa do parâmetro "p". \\
\hline $\begin{array}{l}\text { ASCE-Penman- } \\
\text { Monteith } \\
\text { (ASCE-PM) }\end{array}$ & $\begin{array}{l}\text { Temesgen et al. (2005), } \\
\text { Allen et al. (2006), } \\
\text { Gavilán et al. (2008) }\end{array}$ & - & - & $\begin{array}{l}\text { É um aperfeiçoamento do método PM-FAO, para estimativas da ETo } \\
\text { em escalas de tempo menores que um dia. Os dados de entrada são os } \\
\text { mesmos para PM-FAO, desde que sejam médias do intervalo de tempo. } \\
\text { Normalmente, a escala de tempo é horária. }\end{array}$ \\
\hline $\begin{array}{l}\text { Penman } \\
\text { Modificado (PEN- } \\
\text { MOD) }\end{array}$ & $\begin{array}{l}\text { Doorenbos \& Pruitt } \\
\text { (1977), Yoder et al. } \\
(2005) \text {, Chauhan \& } \\
\text { Shrivastava (2009) }\end{array}$ & & & $\begin{array}{l}\text { Também conhecido por PENMAN FAO 24. É uma modificação do método } \\
\text { PEN. As variáveis são praticamente as mesmas do método PEN. }\end{array}$ \\
\hline \multicolumn{5}{|c|}{$\begin{array}{l}\text { }{ }^{1} \text { Tx, Tn e T: temperaturas diárias máxima, mínima e média, respectivamente; UR: umidade relativa média diária; v: velocidade do vento; n: insolação ou horas de brilho } \\
\text { solar; P: pressão atmosférica média diária; Rs: radiação solar; Rn: saldo de radiação no sistema solo-planta-atmosfera; Tmn: temperatura normal (média de } 30 \text { anos) } \\
\text { do mês ou a média mensal de vários anos com representatividade climática; Tm: temperatura média mensal observada no mês em estudo; ECA: evaporação do tanque } \\
\text { Classe A; URmín: umidade relativa mínima; Tpo: temperatura do ponto de orvalho; G: fluxo de calor no solo. } \\
\text { } 2 \text { Para os métodos que necessitam da radiação solar (Rs) e/ou saldo de radiação (Rn), quando não há a disponibilidade destes elementos medidos, estes poderão ser } \\
\text { facilmente estimados, em função de outros elementos climáticos (Tx, Tn, T e UR), complementados com DJ (dia Juliano) e Lat (latitude). O parâmetro } \lambda \text { (calor latente } \\
\text { de evaporação), presente em vários métodos, equivale a } 2,45 \mathrm{Mj} \mathrm{kg}^{-1} \text {, podendo, também, ser estimado em função de T. É utilizado para converter a radiaçãa à superfície } \\
\text { ou extraterrestre, em equivalente de evaporação. }\end{array}$} \\
\hline
\end{tabular}


da estimativa do saldo de radiação (Rn), quando não se dispõem de medições, o que foi estudado e discutido por Turco et al. (2005), em Jaboticabal (SP), e Yin et al. (2008), na China. Estes autores verificaram que, caso a estimativa de Rn não seja calibrada, a ETo pode ser superestimada em até $27 \%$. Por sua vez, Gavilán et al. (2007), na Espanha, realizaram estudo semelhante, incluindo estimativas e medição de Rn e do fluxo de calor no solo $(\mathrm{G})$, em regiões semiáridas, verificando que estes parâmetros podem apresentar consideráveis diferenças, quando a estimativa da ETo ocorre na escala horária. Já na escala diária, não foram observadas diferenças significativas, considerando-se que as estimativas de Rn e G são confiáveis.

Trajkovic \& Kolakovic (2009), na Croácia e Sérvia, avaliaram cinco métodos de estimativa da ETo, sob condições úmidas. O estudo indicou que o método TC é o mais adequado para estimar a ETo em locais úmidos, quando os dados são insuficientes para aplicar o método PM-FAO. Os demais métodos apresentaram a seguinte ordem decrescente de desempenho: PT, JH, TW e HG.

Para dimensionamento econômico de um sistema de drenagem, na região de Piracicaba, SP (Silva et al. 2005a), embora as estimativas por TW e CAM subestimassem a ETo, em relação à PM-FAO, os autores concluíram que não houve influência dos métodos selecionados, no dimensionamento.

Barreto (2006) e Medeiros (2008), em duas localidades do Estado de São Paulo (Brotas e Jaboticabal, respectivamente), em estudos semelhantes, comparando métodos alternativos para a ETo com PM-FAO, verificaram bons desempenhos atribuídos ao método TW.

Silva et al. (2005b) também avaliaram a eficiência de métodos de estimativa da ETo e concluíram que, na ausência de dados de insolação e velocidade do vento, em Campina Grande (PB), a ETo pode ser substituída, com razoável precisão, pelo método HG. Os métodos LN e TW não apresentaram resultados satisfatórios na estimativa da ETo, em escala diária.

Por sua vez, Tabari (2010) avaliou os métodos de MK, TC, PT e HG, em quatro diferentes tipos climáticos, no Irã. Os resultados mostraram que o método TC teve o melhor desempenho em regiões de clima úmido e frio e em regiões áridas. Contrariamente, o método de MK teve o pior desempenho, exceto para clima frio e úmido. Na condição de clima frio e úmido, o método HG não apresentou bom desempenho. De maneira geral, os métodos TC e HG sobressaíram aos métodos de MK e PT. Maeda et al. (2011), ao sul do Quênia, na África, verificaram que HG foi o método mais apropriado, quando há disponibilidade somente de dados de temperatura do ar. Jabloun \& Sahli (2008) e Gavilán et al. (2008) encontraram alta variabilidade espacial para o método HG, na Tunísia e Espanha, respectivamente. Jabloun \& Sahli (2008) ainda comentam que o método HG superestimou a ETo no interior e subestimou-a na região litorânea, necessitando-se, portanto, ajustar este método, para melhor quantificar a ETo. Na Geórgia, EUA, Suleiman \& Hoogenboom (2007), sob condição de clima úmido, verificaram que o método PT, na escala diária e mensal, subestimou a ETo, durante o inverno, ao centro e sudeste do Estado, e superestimou-a no verão, para a região costeira e áreas montanhosas.

Reis et al. (2007) realizaram estudo comparativo de métodos empíricos, para estimativa da ETo, em comparação ao método universal padrão PM-FAO, para as condições climáticas dos municípios de Sooretama, Cachoeiro de Itapemirim e Venda Nova do Imigrante, localizados, respectivamente, nas regiões norte, sul e serrana do Estado do Espírito Santo. Para as condições climáticas das localidades onde se realizou o trabalho, para o período seco, os métodos que apresentaram as melhores estimativas acompanharam a seguinte ordem decrescente: PEN, PT, PEN-MOD, BC, TC, RS e MK.

Para a bacia hidrográfica do Ribeirão Lavrinha, região da Serra da Mantiqueira, em Minas Gerais, Pereira et al. (2009) compararam diversos métodos com o PM-FAO, nas escalas diária e mensal, durante os períodos chuvoso (outubro a março) e seco (abril a setembro). Os métodos de JH, RS, PEN e BC foram adequados para estimar a ETo, em escala diária, independentemente da época do ano, enquanto, em escala mensal, o método JH foi o que apresentou melhor ajuste, seguido dos métodos KF modificado, RS e HM modificado.

Tagliaferre et al. (2010) avaliaram o desempenho dos métodos empíricos PEN-MOD, RS, BC, HG, PT e TC, em relação ao método padrão PM-FAO, para as condições climáticas do sul da Bahia, no Município de Eunápolis, cujo clima é classificado como subúmido. Os métodos BC e RS apresentaram melhor desempenho para estimativa de ETo, em quatro escalas de tempo: diária, três, cinco e sete dias. Os métodos PT e TC subestimaram os valores de ETo obtidos pelo método padrão PM-FAO. O método PEN-MOD subestimou a ETo, para valores 
inferiores a $3 \mathrm{~mm} \mathrm{~d}^{-1}$, e superestimou-a para valores maiores. O método HG se destacou como o pior método utilizado para estimar a ETo.

De acordo com estudos de Syperreck et al. (2008), em Palotina, Estado do Paraná, os métodos HG, TW e CAM apresentaram bom desempenho, quando comparados ao PM-FAO.

Borges \& Mendiondo (2007) verificaram a precisão dos métodos CAM, BC, HM, HG, TW e KF, em comparação ao método PM-FAO, para a bacia do Rio Jacupiranga (SP). Os resultados obtidos indicaram que, na região, os métodos $\mathrm{HG}$ e CAM podem ser aplicados tanto na forma original como na formulação modificada. Segundo estes autores, embora tenha apresentado boa correlação, o método original de Kharrufa (KF) teve baixo índice de confiança. Com o uso das constantes e parâmetros recalibrados para a região, a confiabilidade aumentou para todos os métodos e a aplicabilidade local foi verificada com a seguinte ordem de confiabilidade: HG, CAM, KF, HM, BC e TW. O método CAM, que é um método simplificado, com base no de Thornthwaite (TW), para regiões com temperatura positiva, apresentou altos índices de confiabilidade, em todas as situações. Devido à alta confiabilidade, sugere-se o uso do método HG modificado, para a região da bacia do Rio Jacupiranga, visto que este necessita somente de dados de temperatura do ar, conjugando simplicidade e exequibilidade.

Adeboye et al. (2009) avaliaram o desempenho dos métodos JH e HG, comparando-os com o PM-FAO, na bacia do Rio Ogun-Osun, na Nigéria. Concluíram que o método JH é recomendado para o cálculo da ETo em situações em que apenas as temperaturas máximas e mínimas estão disponíveis.

Yoder et al. 2005, em estudo para a região úmida do platô de Cumberland, abrangendo os Estados do Alabama, Kentucky, Virgínia e Tennessee, nos Estados Unidos, testaram vários métodos, comparando-os com dados de lisímetro de pesagem. O método PM-FAO comprovou sua consistência na estimativa da ETo, seguido pelos métodos PEN e TC. Os métodos PEN-MOD e PT superestimaram a ETo, enquanto o método MK subestimou-a. Os resultados para HG apresentaram baixa correlação com os dados do lisímetro. Os autores consideraram que o método TC pode ser uma alternativa satisfatória, por requerer menos dados de entrada, ou seja, somente a temperatura média do ar e os dados de radiação solar.

Chauhan \& Shrivastava (2009) também compararam os métodos de BC, RS, PEN-MOD e
TCA com o método padrão PM-FAO e os resultados levaram à conclusão de que o método $\mathrm{BC}$ apresentou o melhor desempenho, seguido dos métodos TCA, PEN-MOD e RS, com eficiências de $83 \%$, $80 \%, 65,5 \%$ e $53,8 \%$, respectivamente. Este estudo foi realizado em Raipur (Chhattisgarh), na Índia. Nazeer (2010), no Paquistão, estudou o desempenho dos métodos HG, PEN e PM-FAO, comparando-os com o método TCA, e verificou que o PM-FAO foi altamente correlacionado com o TCA, seguido pelo HG e, por fim, pelo PEN.

Barros et al. (2009), para a região de Seropédica (RJ), correlacionou medidas obtidas em lisímetros de pesagem com os métodos PM-FAO, HG, CAM, PT, MK e TCA. As melhores estimativas de ETo foram obtidas com os métodos PM-FAO, TCA e HG, principalmente para períodos de 3 e 5 dias. Os métodos CAM, MK e PT não estimaram satisfatoriamente a ETo, para observações agrupadas em pequenos períodos, por isto, foram recomendados para escalas temporais maiores que cinco dias.

López-Urrea et al. (2006) avaliaram sete métodos de cálculo diário de ETo, em comparação a um lisímetro de pesagem, para um clima semiárido, na Província de Albacete, na Espanha. Para as condições de semiaridez, os autores concluíram que o método PM-FAO foi o mais adequado para o cálculo da ETo. O método HG foi o segundo mais preciso, apesar de sua simplicidade, juntamente com o método RS, superestimando a ETo em torno de 5\%. O método PEN-MOD e, especialmente, o método BC superestimaram-na significativamente, enquanto $\mathrm{O}$ método PEN subestimou-a em 30\%.

$\mathrm{Xu} \&$ Chen (2005) avaliaram sete métodos de evapotranspiração, dentre eles, especificamente para a ETo, o TW, HG, MK e PT, comparando-os com dados de lisímetros de pesagem, na Alemanha. Os autores concluíram que TW, MK e PT obtiveram igualmente bons resultados, com erros abaixo de $10 \%$. O pior resultado foi obtido com o método HG.

Er-Raki et al. (2010), sob condições semiáridas, na bacia Tensift (centro de Marrocos) e no Vale Yaqui (noroeste do México), identificaram melhores resultados para o método HG. Por outro lado, o desempenho dos métodos PT e MK foram piores, exceto sob condições úmidas. No entanto, os autores sugerem que parâmetros específicos, nos métodos PT e MK, quando calibrados localmente, melhoram bastante o desempenho destes. 
Vescove \& Turco (2005), ao analisarem, para Araraquara (SP), nos períodos verão-outono e inverno-primavera, os métodos PM-FAO, TCA, RS e MK, observaram que o método MK subestimou a ETo, no período inverno-primavera, mais que no outro período. Com relação ao método RS, para o período verão-outono, houve superestimativa pelo método mais do que no período inverno-primavera. $\mathrm{O}$ método TCA superestimou a ETo em $26 \%$, no período verão-outono, e $24 \%$, no período inverno-primavera, em relação ao método-padrão PM-FAO.

Para o Estado de Sergipe, Sousa et al. (2010) estimaram a ETo com base nos métodos TCA, RS, HG e LN, comparando-os com o método PM-FAO, e os melhores desempenhos foram obtidos com os métodos que utilizam a radiação solar como elemento de entrada. O método TCA não apresentou desempenho satisfatório em nenhum perímetro de estudo.

Vega \& Jara (2009), no Equador, em duas localidades de altitudes diferentes, procuraram ajustar os métodos HD e HG, com três modificações em cada um deles, com o propósito de se estimar a ETo, para período de três dias. As comparações foram efetuadas com o método TCA e eles concluíram que a qualidade das estimativas da ETo, com as três modificações de ambos os métodos, foram melhores, em comparação com os resultados da ETo obtidos por PM-FAO.

Cinco equações de estimativa de coeficiente de tanque (Kp) (propostas por Cuenca 1989, Snyder 1992, Pereira et al. 1995, Orang 1998, Raghuwanshi \& Wallender 1998 apud Gundekar et al. 2008), para ajuste da evaporação do Tanque Classe A, na obtenção da ETo, foram avaliadas para uma região semiárida, na Índia, para cálculo da ETo, e, comparando-a com a estimada por PM-FAO, verificou-se que a equação de Snyder foi a mais adequada.

Sabziparvar et al. (2010), em dois tipos de climas, no Irã, analisaram sete diferentes métodos para estimativa do coeficiente de tanque (Kp), para o ajuste da evaporação do Tanque Classe A, na estimativa da ETo. A maioria das equações de Kp não apresentou bons resultados, exceto para as equações de Raghuwanshi-Wallender, em clima semiárido; de Snyder, para climas áridos e quentes; e para a equação de Orang, em ambos os climas. Trajkovic \& Kolakovic (2010), em estudo semelhante, encontraram melhor desempenho para a equação de Snyder, na região de Policoro, Itália.

\section{CONSIDERAÇÕES FINAIS}

Embora a evapotranspiração, sob o aspecto conceitual, possa ser relativamente simples, quando se parte para os aspectos práticos de sua medição e estimativa, ainda nota-se a dificuldade e complexidade em apresentar uma solução que seja plenamente satisfatória e, portanto, continuam surgindo constantes estudos, visando a dar uma resposta mais consistente ao problema.

Neste sentido, o método de Penman-Monteith-FAO (PM-FAO) surgiu em 1990, como uma nova proposta para estimar a ETo, revolucionando os estudos sobre o assunto. Pelos estudos revisados neste trabalho, são raríssimas as regiões em que este método não seja recomendado.

Embora o método PM-FAO seja amplamente divulgado e recomendado, ainda é possível encontrar extensões deste método, com o intuito de aperfeiçoamento, ou para atender a uma questão crucial, envolvendo elementos meteorológicos nem sempre disponíveis, devido à limitação destes. Até mesmo a forma de se estimar o saldo de radiação (Rn) e o fluxo de calor no solo $(\mathrm{G})$ leva a estimativas diferenciadas. Com a difusão de estações meteorológicas automáticas, tem-se verificado que a ETo por PM-FAO pode ser estimada em escala horária, contudo, maior atenção deve ser destinada às medições de $\mathrm{Rn}$ e $\mathrm{G}$, que variam significativamente durante o dia.

Quanto ao desenvolvimento do balanço hídrico climatológico proposto por Thornthwaite \& Mather (1955), presente em quase todos os estudos de zoneamento agroclimático, apesar de em sua proposição a evapotranspiração potencial ser estimada pelo método de Thornthwaite (TW), no Brasil, já existem alguns estudos aplicando o método PM-FAO, o que, de fato, caracteriza melhor a demanda de evapotranspiração, pois, sob diferentes condições climáticas, o método TW nem sempre se correlaciona bem com o método padrão PM-FAO.

Havendo a indisponibilidade de dados de entrada, para o método PM-FAO, justifica-se a utilização de métodos alternativos. Verifica-se, na maioria dos trabalhos presentes na literatura, que os métodos mais estudados são o TW, HG, BC, PT, TC, MK, LN, HM, HD, RS, JH, TCA e, evidentemente, o método PM-FAO. Diferentes métodos são desenvolvidos ou recomendados para diferentes condições climáticas, dependendo da disponibilidade de dados locais, precisão exigida e/ou escala temporal. Contudo, para 
adequá-los a uma condição específica, muitos deles passam por ajustes (calibrações), evidenciando-se, portanto, que, para a adoção de um método, deve-se atentar para as condições e/ou semelhanças climáticas em que ele foi desenvolvido.

O presente estudo propôs apresentar os trabalhos mais recentes sobre desempenhos de métodos de estimativa da ETo. Pode-se concluir que, quando há disponibilidade de dados para o método PM-FAO, este deve ser o recomendado como padrão, tendo em vista sua comprovada precisão, independentemente da escala temporal e espacial. Caso contrário, deve-se averiguar um método que esteja de acordo com os dados disponíveis e se é adequado às condições climáticas do local. Em resumo, estes são alguns dos motivos pelos quais diversos trabalhos de pesquisa procuram alternativas que viabilizem a estimativa da ETo, e somente por meio destes é que se poderá dar uma orientação de resposta à pergunta levantada inicialmente: "Qual o melhor método a ser aplicado?".

Finalmente, ao se utilizarem coeficientes de culturas $(\mathrm{Kc})$, para estimativa da evapotranspiração de uma cultura qualquer (ETc), é importante identificar a origem de obtenção do Kc. Recomenda-se que a estimativa da ETo siga o mesmo método e critérios adotados originalmente na determinação do Kc.

\section{REFERÊNCIAS}

ADEBOYE, O. A. et al. Evaluation of FAO-56 PenmanMonteith and temperature based models in estimating reference evapotranspiration using complete and limited data, application to Nigeria. Agricultural Engineering International, Beijing, v. 11, n. 1, p. 1-25, out. 2009.

ALLEN, R. G. et al. A recommendation on standardized surface resistance for hourly calculation of reference ETo by the FAO56 Penman-Monteith method. Agricultural Water Management, Amsterdam, v. 81, n. 1, p. 1-22, 2006.

ALLEN, R. G. et al. Crop evapotranspiration: guidelines for computing crop water requirements. Rome: FAO, 1998. (Irrigation and drainage paper, 56).

BARRETO, C. E. A. G. Balanço hídrico em zona de afloramento do sistema aquifero Guarani a partir de monitoramento hidrogeológico em bacia representativa. 2005. 249 f. Dissertação (Mestrado em Hidráulica e Sanemento)-Escola de Engenharia de São Carlos, Universidade de São Paulo, São Carlos, 2006.

BARROS, V. R. et al. Avaliação da evapotranspiração de referência na região de Seropédica, Rio de Janeiro, utilizando lisímetro de pesagem e modelos matemáticos.
Revista Brasileira de Ciências Agrárias, Recife, v. 4, n. 2, p. 198-203, abr./jun. 2009.

BEZERRA, B. G. de; SILVA, B. B. de; FERREIRA, N. J. Estimativa da evapotranspiração real diária utilizando-se imagens digitais TM - Landsat 5. Revista Brasileira de Meteorologia, São José dos Campos, v. 23, n. 3, p. 305317, 2008.

BORGES, A. C. de; MENDIONDO, E. M. Comparação entre equações empíricas para estimativa da evapotranspiração de referência na Bacia do Rio Jacupiranga. Revista Brasileira de Engenharia Agrícola e Ambiental, Campina Grande, v. 11, n. 3, p. 293-300, 2007.

CAMARGO, A. P.; CAMARGO, M. B. P. Uma revisão analítica da evapotranspiração potencial. Bragantia, Campinas, v. 59, n. 2, p. 125-137, 2000.

CARVALHO, L. G. de et al. Clima. In: SCOLFORO, J. R.; CARVALHO, L. M. T. de; OLIVEIRA, A. D. de. Zoneamento ecológico-econômico do Estado de Minas Gerais: componentes geofísico e biótico. Lavras: UFLa, 2008. p. 89-102.

CHAUHAN, S.; SHRIVASTAVA, R. K. Performance evaluation of reference evapotranspiration estimation using climate based methods and artificial neural networks. Water Resource Management, New York, v. 23, n. 5, p. 825-837, 2009.

DOORENBOS, J.; PRUITT, W. O. Crop water requirements. Rome: FAO, 1977. (Irrigation and drainage paper, 24).

ER-RAKI, S. et al. Assessment of reference evapotranspiration methods in semi-arid regions: can weather forecast data be used as alternate of ground meteorological parameters? Journal of Arid Environments, Amsterdam, v. 74, n. 12, p. 1587-1596, 2010.

FOLHES, M. T.; RENNÓ, C. D.; SOARES, J. V. Remote sensing for irrigation water management in the semi-arid Northeast of Brazil. Agricultural Water Management, Amsterdam, v. 96, n. 10, p. 1398-1408, 2009.

GAVILÁn, P.; BERENGENA, J.; ALLEN, R. G. Measuring versus estimating net radiation and soil heat flux: impact on Penman-Monteith reference ET estimates in semiarid regions. Agricultural Water Management, Amsterdam, v. 89, n. 3, p. 275-286, 2007.

GAVILÁN, P.; ESTÉVEZ, J. de; BERENGENA, J. Comparison of standardized reference evapotranspiration equations in southern Spain. Journal of Irrigation and Drainage Engineering, New York, v. 134, n. 1, p. 1-12, jan./fev. 2008.

GUNDEKAR, H. G. et al. Evaluation of pan coefficient for reference crop evapotranspiration for semi-arid region. Irrigation Science, Sidney, v. 26, n. 2, p. 169-175, 2008. 
JABLOUN, M. de; SAHLI, A. Evaluation of FAO-56 methodology for estimating reference evapotranspiration using limited climatic data application to Tunisia. Agricultural Water Management, Amsterdam, v. 95, n. 6, p. 707-715, 2008.

JENSEN, M. E.; BURMAN, R. D.; ALLEN, R. G. Evapotranspiration and irrigation water requirements. New York: ASCE, 1990. (Manuals and reports on engineering practice, n. 70).

KUMAR, M.; RAGHUWANSHI, N. S.; SINGH, R. Artificial neural networks approach in evapotranspiration modeling: a review. Irrigation Science, Sidney, v. 29, n. 1, p. 11-25, 2010.

LÓPEZ-URREA, R. et al. Testing evapotranspiration equations using lysimeter observations in a semiarid climate. Agricultural Water Management, Amsterdam, v. 85, n. 1, p. 15-26, 2006.

MAEDA, E. E.; WIBERG, D. A.; PELLIKKA, P. K. E. Estimating reference evapotranspiration using remote sensing and empirical models in a region with limited ground data availability in Kenya. Applied Geography, Amsterdam, v. 31, n. 1, p. 251-258, 2011.

MEDEIROS, P. V. Análise da evapotranspiração de referência a partir de medidas lisimétricas e ajuste estatístico de estimativas de nove equações empiricoteóricas com base na equação de Penman-Monteith. 2008. 241 f. Dissertação (Mestrado em Hidráulica e Saneamento)-Escola de Engenharia de São Carlos, Universidade de São Paulo, São Carlos, 2008.

NAZEER, M. Comparison of different methods for estimation of potential evapotranspiration. The Nucleus, Peshawar, v. 47, n. 1, p. 41-46, 2010.

PENMAN, H. L. Natural evaporation from open water, bare soil, and grass. Proceedings of the Royal Society, London, v. 193, n. 1, p. 120-146, 1948.

PEREIRA, A. R.; VILLA NOVA, N. A.; SEDIYAMA. G. C. Evapo(transpi)ração. Piracicaba: Fealq, 1997.

PEREIRA, D. dos R. et al. Desempenho de métodos de estimativa da evapotranspiração de referência para a região da Serra da Mantiqueira, MG. Ciência Rural, Santa Maria, v. 39, n. 9, p. 2488-2493, dez. 2009.

REIS, E. F. de; BRAGANÇA, R. de; GARCIA, G. O. Estudo comparativo da estimativa da evapotranspiração de referência para três localidades do Estado do Espírito Santo no período seco. Idesia, Arica, v. 25, n. 3, p. 75-7854, 2007.

SABZIPARVAR, A. A. et al. Evaluation of class A pan coefficient models for estimation of reference crop evapotranspiration in cold semi-arid and warm arid climates. Water Resouce Management, Amsterdam, v. 24, n. 5, p. 909-920, 2010.
SEDIYAMA, G. C. Estimativa da evapotranspiração: histórico, evolução e análise crítica. Revista Brasileira de Agrometeorologia, Santa Maria, v. 4, n. 1, p. 1-7, 1996.

SENTElHAS, P. C.; GILlESPIE, T. J.; SANTOS, E. A. Evaluation of FAO Penman-Monteith and alternative methods for estimating reference evapotranspiration with missing data in Southern Ontario, Canada. Agricultural Water Management, Amsterdam, v. 97, n. 5, p. 635-644, 2010.

SENTELHAS, P. C.; SANTOS, D. L. dos; MACHADO, R. E. Water deficit and water surplus maps for Brazil, based on FAO Penman-Monteith potential evapotranspiration. Engenharia Agrícola, Jaboticabal, v. 25, n. 3, p. 713-721, set./dez. 2008.

SILVA, B. M. et al. Estimate of reference evapotranspiration by FAO Penman-Monteith equation only having maximum and minimum temperatures in Lavras, Minas Gerais State, Brazil. In: CONGRESSO BRASILEIRO DE ENGENHARIA AGRÍCOLA, 37., Foz do Iguaçu. Anais... Foz do Iguaçu: SBEA, 2008. 1 CD-ROM.

SILVA, K. O. et al. Análise de métodos de estimativa de evapotranspiração na otimização de sistemas de drenagem. Revista Brasileira de Engenharia Agrícola e Ambiental, Campina Grande, v. 9, n. 2, p. 161-165, 2005a.

SILVA, V. P. R. et al. Desenvolvimento de um sistema de estimativa da evapotranspiração de referência. Revista Brasileira de Engenharia Agrícola e Ambiental, Campina Grande, v. 9, n. 4, p. 547-553, 2005 b.

SOUSA, I. F. et al. Evapotranspiração de referência nos perímetros irrigados do Estado de Sergipe. Revista Brasileira de Engenharia Agrícola e Ambiental, Campina Grande, v. 14, n. 6, p. 633-644, 2010.

SULEIMAN, A. A.; HOOGENBOOM, G. A comparison of ASCE and FAO-56 reference evapotranspiration for a 15-min. time step in humid climate conditions. Journal of Hydrology, Amsterdam, v. 375, n. 3/4, p. 326-333, set. 2009.

SULEIMAN, A. A.; HOOGENBOOM, G. Comparison of Priestley-Taylor and FAO-56 Penman-Monteith for daily reference evapotranspiration estimation in Georgia. Journal Irrigation and Drainage Engineering, New York, v. 133, n. 2, p. 175-182, mar./abr. 2007.

SYPERRECK, V. L. G. et al. Avaliação de desempenho de métodos para estimativas de evapotranspiração de referência para a região de Palotina, Estado do Paraná. Acta Scientiarum Agronomy, Maringá, v. 30, n. 5, p. 603609, 2008.

TABARI, H. Evaluation of reference crop evapotranspiration equations in various climates. Water Resource Management, Amsterdam, v. 24, n. 10, p. 23112337, 2010. 
TAGLIAFERRE, C. et al. Estudo comparativo de diferentes metodologias para determinação da evapotranspiração de referência em Eunápolis - BA. Revista Caatinga, Mossoró, v. 23, n. 1, p. 103-111, jan./mar. 2010.

TEMESGEN, B. et al. Comparison of some reference evapotranspiration equations for California. Journal of Irrigation and Drainage Engineering, New York, v. 131, n. 1, p. 73-84, jan./fev. 2005.

THORNTHWAITE, C. W. An approach towards a rational classification of climate. Geographycal Review, London, v. 38, n. 1, p. 55-94, jan. 1948.

THORNTHWAITE, C. W.; MATHER, J. R. The water balance: publications in climatology. New Jersey: Drexel Institute of Technology, 1955.

THORNTHWAITE, C. W.; WILM, H. G. Report of the committee on evapotranspiration and transpiration, 19431944. Transactions of the American Geophysical Union, Washington, DC, v. 25, n. 5, p. 686-693, 1944.

TRAJKOVIC, S.; KOLAKOVIC, S. Comparison of simplified pan-based equations for estimating reference evapotranspiration. Journal of Irrigation and Drainage Engineering, New York, v. 136, n. 2, p. 137-140, fev. 2010.

TRAJKOVIC, S.; KOLAKOVIC, S. Evaluation of reference evapotranspiration equations under humid conditions. Water Resource Management, Amsterdam, v. 23, n. 14, p. 3057-3067, 2009.
TURCO, J. E. P. de; FARIA, M. T. de; FERNANDES, E. J. Influência da forma de obtenção do saldo de radiação na comparação de métodos de estimativa da evapotranspiração de referência. Irriga, Botucatu, v. 10, n. 3, p. 215-228, ago./out. 2005.

VEGA, E. C.; JARA, J. C. Estimación de la evapotranspiración de referencia para dos zonas (costa y región andina) del Ecuador. Engenharia Agrícola, Jaboticabal, v. 29, n. 3, p. 390-403, jul./set. 2009.

VESCOVE, H. V.; TURCO, J. E. P. Comparação de três métodos de estimativa da evapotranspiração de referência para a região de Araraquara - SP. Engenharia Agrícola, Jaboticabal, v. 25, n. 3, p. 713-721, set./dez. 2005.

XU, C-Y.; CHEN, D. Comparison of seven models for estimation of evapotranspiration and groundwater recharge using lysimeter measurement data in Germany. Hydrological Processes, Chichester, v. 19, n. 18, p. 3717 3734, 2005.

YIN, Y. et al. Radiation calibration of FAO56 Penman-Monteith model to estimate reference crop evapotranspiration in China. Agricultural Water Management, Amsterdam, v. 95, n. 1, p. 77-84, 2008.

YODER, R. E.; ODHIAMBO, L. O.; WRIGHT, W. C. Evaluation of methods for estimating daily reference crop evapotranspiration at a site in the humid Southeast United States. Applied Engineering in Agriculture, St. Joseph, v. 21, n. 2, p. 197-202, 2005. 